\title{
Osteosarcoma extraesquelético retroperitoneal en un perro: reporte de un caso
}

\section{Retroperitoneal extraskeletal osteosarcoma in a dog: a case report}

\section{César Piedra-Mora ${ }^{1}$, Daniel Barrantes-Murillo ${ }^{1}$, Alejandro Alfaro-Alarcón ${ }^{1} \bowtie$.}

1 Departamento de Patología, Escuela de Medicina Veterinaria, Universidad Nacional, Costa Rica. cespiedram@gmail.com; danielfelipebarrantesmurillo@gmail.com; alejandro.alfaro.alarcon@una.cr

Recibido: 28 de Octubre de 2015. Corregido: 5 de Mayo de 2016. Aceptado: 6 de Mayo de 2016.

Resumen: Se presentó un perro, de raza mixta, no castrado, 15 años de edad, con hiporexia, constipación, letargiay dolor abdominal. Enelexamen físico, seidentificó una masa intraabdominal. Las pruebas hematológicas evidenciaron anemia leve, normocítica y normocrómica y leucocitosis a causa de neutrofilia. Los resultados de bioquímica sérica mostraron alteraciones del nitrógeno ureico sanguíneo, fosfatasa alcalina sérica y creatinina. En el ultrasonido, se detectó un nódulo en bazo. Las radiografías evidenciaron un área radiopaca en abdomen, cerca de las vértebras lumbares. Se realizó laparotomía exploratoria y se encontró dos masas retroperitoneales. Se realizó la eutanasia del paciente, debido al difícil abordaje quirúrgico. El análisis histopatológico e inmunohistoquímico de las masas fue concluyente de un osteosarcoma extraesquelético. Los osteosarcomas extraesqueléticos son tumores malignos, de origen mesenquimal infrecuentes, sin involucramiento primario de tejido óseo o periostio. La localización retroperitoneal de estos tumores pocas veces ha sido reportada.

Palabras clave: osteosarcoma extraesquelético, retroperitoneo, perro.

Abstract: A 15-year-old not neutered mixed-breed dog was presented with hyporexia, constipation, lethargy and abdominal pain. An intra abdominal mass was identified during the physical examination. Mild normocytic and normochromic anemia and leukocytosis due to neutrophilia were detected in the complete blood count. Serum biochemistry results showed alterations in blood urea nitrogen, serum alkaline phosphatase, and creatinine. A splenic nodule was detected during the ultrasonographic examination, and radiographic findings showed a radiopaque area in the abdomen near the lumbar vertebrae. An exploratory laparotomy was done, and two retroperitoneal masses were found. Due to the difficult surgical approach euthanasia was elected. Histopathological and immunohistochemical evaluation of tissues was performed and the diagnosis of extraskeletal osteosarcoma was made. Extraskeletal osteosarcoma is an infrequent malign mesenchymal tumor without primary involvement of bone tissue or periosteum. Seldom has the retroperitoneal localization of this tumor been reported.

Keywords: Extraskeletal osteosarcoma, retroperitoneum, dog. 


\section{Introduccción}

El osteosarcoma extraesquelético (OEE) es una neoplasia maligna, poco común, de origen mesenquimal. Se caracteriza por la formación de tejido osteoide en órganos viscerales y tejidos blandos, sin que exista relación con tejido óseo o periostial (Patnaik 1990; Langenbach et al. 1998; Spugnini et al. 2001; van de Sant et al. 2004; Urbiztondo et al. 2010; Mackenzie et al. 2012; Marcus et al. 2012; Timian et al. 2012). Representan cerca del 1\% de los osteosarcomas (Woldemeskel \& Grice 2011; Timian et al. 2012). Han sido descritos en el tracto gastrointestinal, yeyuno, esófago, retroperitoneo, pulmones, tracto respiratorio, sacos anales, tejido subcutáneo, bazo, tracto urinario, hígado, músculo, piel, ojo y glándula mamaria (van de Sant et al. 2004; Timian et al. 2011; Woldemeskel \& Grice 2011; Marcus et al. 2012; Duffy et al. 2015). También se han reportado en tiroides, glándulas adrenales, glándulas salivales, meninges, corazón, ovarios, testículos, tejidos renales y raíz mesentérica (Duffy et al. 2015). Aproximadamente, un $70 \%$ de los OEE se origina en glándula mamaria; los restantes, en su mayoría, se ubican en la cavidad abdominal (Urbiztondo et al. 2010).

Los criterios establecidos para clasificar un OEE, son: 1) el tumor debe tener un patrón uniforme sarcomatoso, el cual excluya la posibilidad de que sea un tumor mesenquimal mixto; 2) el tumor debe producir osteoide o hueso; 3) debe tener un índice mitótico alto; 4) se debe excluir cualquier tipo de relación anatómica al tejido óseo o de periostio (Kuntz et al. 1998; Spugnini et al. 2001; Urbiztondo et al. 2010).

El OEE es un tumor descrito en perros de 9 a 11 años de edad (Patnaik 1990; Kuntz et al. 1998; Langenbach et al. 1998; Duffy et al. 2015), sin predilección de sexo (Patnaik 1990; Kuntz et al. 1998; Langenbach et al. 1998; Duffy et al. 2015), tampoco de raza (Patnaik 1990; Kuntz et al. 1998; Urbiztondo et al. 2011), la metástasis es común, ocurre en un $64 \%$ de los casos y, usualmente, en los pulmones (Kuntz et al. 1998; Woldemeskel \& Grice 2011); con un tiempo de sobrevivencia que va de 23 a 33 días sin tratamiento (Patnaik 1990; Kuntz et al. 1998; Urbiztondo et al. 2010; Timian et al. 2011; Mackenzie et al. 2012; Marcus et al 2012).

\section{Reporte de caso}

Se presentó, en el Hospital de Especies Menores y Silvestres de la Universidad Nacional, un perro macho entero, de 15 años de edad, raza mixta, de $8.5 \mathrm{~kg}$ de peso, con historia de constipación de 4 días e hiporexia desde hacía aproximadamente 2 semanas. El examen físico presentaba dolor en abdomen medio, en relación con una masa de consistencia dura a la palpación. No se encontró alteraciones musculo-esqueléticas. El paciente no recibió tratamiento previo a la evaluación física. Dentro de anormalidades del hemograma, se encontró anemia leve normocítica, normocrómica, no regenerativa (hematocrito de $29.6 \%$; rango de referencia $37-55 \%)$; y leucocitosis leve $\left(17.74 \times 10^{3}\right.$ células $/ \mu \mathrm{l} ; 6-16 \times 10^{3}$ células $\left./ \mu \mathrm{l}\right)$

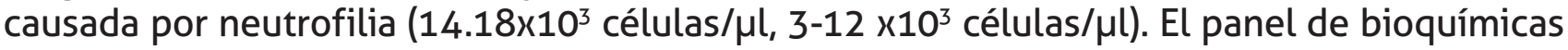
séricas mostró elevación del nitrógeno ureico $(46 \mathrm{mg} / \mathrm{dl} ; 7-25 \mathrm{mg} / \mathrm{dl})$, de creatinina $(2.5$ $\mathrm{mg} / \mathrm{dl} ; 0.3-1.4 \mathrm{mg} / \mathrm{dl}$ ) y de fosfatasa alcalina sérica (FAS) (898 UI/L, 20-150 UI/L) (Cuadro 1). La evaluación ultrasonográfica del abdomen reveló la presencia de un nódulo en bazo de 
aproximadamente $1.4 \mathrm{~cm}$ de diámetro; un aumento de ecogenicidad y disminución de la relación cortico-medular del riñón izquierdo y derecho; además, presencia de una estructura quística de aproximadamente $3 \mathrm{~cm}$ de diámetro en el polo craneal del riñón izquierdo; también, la presencia de gran cantidad de heces en colon, que imposibilitaba la evaluación completa del abdomen. La evaluación radiográfica del abdomen se realizó con vistas ortogonales y evidenció la presencia de un área radiopaca con mineralización irregular amorfa, localizada en el abdomen medio, en el plano correspondiente a las vértebras lumbares L4, L5 y L6. Con base en estos hallazgos, se realizó una laparotomía exploratoria, en la cual se encontró dos masas en el espacio retroperitoneal, de consistencia dura, adheridas por tejido fibroso a la pared dorsal, sin asociación a órganos abdominales ni a estructuras óseas. En el bazo se notó la presencia de un nódulo, previamente descrito durante la evaluación ultrasonográfica. Durante la cirugía, el propietario fue contactado. Se optó por la eutanasia con pentobarbital sódico y difenilhidantoína sódica intravenosa. Posteriormente, se removió las masas y el bazo, las cuales fueron remitidas al laboratorio de patología. No se realizó necropsia, por decisión del propietario. Los tejidos se fijaron en formalina buferada al $10 \%$ y procesados de forma rutinaria para su análisis histopatológico (Miller 2016).

Cuadro 1: Valores de hemograma y químicas séricas del paciente.

\begin{tabular}{ccc}
\hline Parámetro & Valores & Referencia \\
\hline $\begin{array}{c}\text { Hematocrito } \\
\text { Leucocitos }\end{array}$ & $26.9 \%$ & $37-55 \%$ \\
Neutrófilos & $17.74 \times 10^{3}$ células $/ \mu \mathrm{l}$ & $6-16 \times 10^{3}$ células $/ \mu \mathrm{l}$ \\
BUN & $14.18 \times 10^{3}$ células $/ \mu \mathrm{l}$ & $3-12 \times 10^{3}$ células $/ \mu \mathrm{L}$ \\
Creatinina & $46 \mathrm{mg} / \mathrm{dl}$ & $7-25 \mathrm{mg} / \mathrm{dl}$ \\
FAS & $2.5 \mathrm{mg} / \mathrm{dl}$ & $0.3-1.4 \mathrm{mg} / \mathrm{dl}$ \\
\hline
\end{tabular}

Una de las masas presentaba un tamaño aproximado de $9 \mathrm{~cm}$ de diámetro, color blanco, aspecto marmoleado, forma irregular, bien circunscrita, multinodular (Figura $1 \mathrm{~A}$ ). La segunda masa presentaba las mismas características macroscópicas, con un diámetro 3,5 cm (Figura $1 \mathrm{~B}$ ). El bazo presentaba un nódulo de $1,5 \mathrm{~cm}$ de diámetro, crecimiento exofítico, cuya superficie de corte es multinodular, de color café a blanco, bien delimitado, no encapsulado (Figura $1 \mathrm{C}$ ). Al corte, la masa de $9 \mathrm{~cm}$, blanca, con pequeñas áreas hemorrágicas, no encapsulada, de forma irregular y consistencia dura (Figura 2).

Histopatológicamente, las dos masas retroperitoneales retroperitoneales presentaban el mismo tipo de proliferación neoplásica. El tejido neoplásico proliferaba en forma sólida (Figura $3 \mathrm{~A}$ ), en un muy escaso estroma fibrovascular, con formación multifocal de tejido 
osteoide (Figura 3 B), en asociación con los osteoblastos neoplásicos, así como pequeños focos de mineralización irregular. Las células neoplásicas presentaban núcleos ovalados a poliédricos, en algunos casos, fusiformes, con cromatina granular, 1 a 2 nucléolos pequeños basofílicos prominentes, una figura mitótica por campo de $40 \mathrm{x}$ y escaso citoplasma eosinofílico. Las células tenían un moderado pleomorfismo, con moderada anisocariosis y anisocitosis. El bazo presentaba una marcada hiperplasia de la pulpa blanca, caracterizada por una población monomórfica de linfocitos pequeños y medianos, con cromatina condensada y escaso citoplasma, alrededor de las arteriolas del parénquima esplénico, sin presencia de tejido neoplásico. Esto corresponde a hiperplasia folicular esplénica. Las características morfológicas de la proliferación neoplásica maligna de origen mesenquimal, son concluyentes de un osteosarcoma osteoblástico extraesquelético.

Adicionalmente, el tejido neoplásico fue caracterizado por medio de inmunohistoquímica (IHO) para evaluar la expresión de citoqueratina (Citoqueratina monoclonal de ratón antihumano, 1:400, Dako) (Figura 4 A) y vimentina (Monoclonal de ratón Anti-Vimentina, 1:200, Dako) (Figura 4 B). El tumor analizado, en este caso, presentó únicamente una marcada expresión de vimentina, fue negativo para citoqueratina.

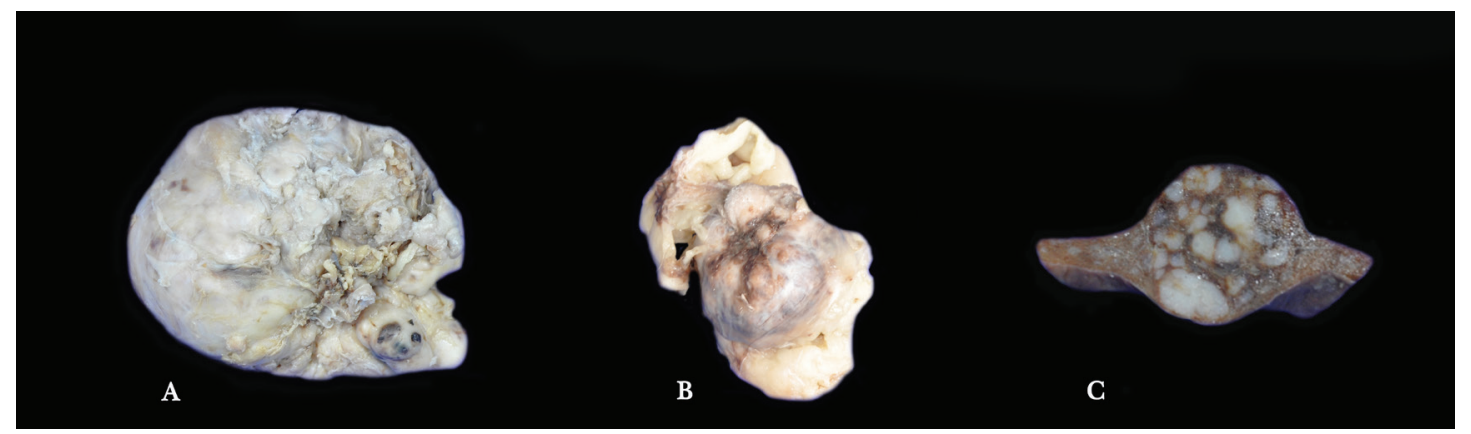

Figura 1. Osteosarcoma extraesquelético retroperitoneal, aspecto macroscópico externo de los tumores, los cuales son irregulares, no encapsulados y de consistencia dura (A y B), corte transversal de un segmento de bazo con hiperplasia folicular linfoide (C). 


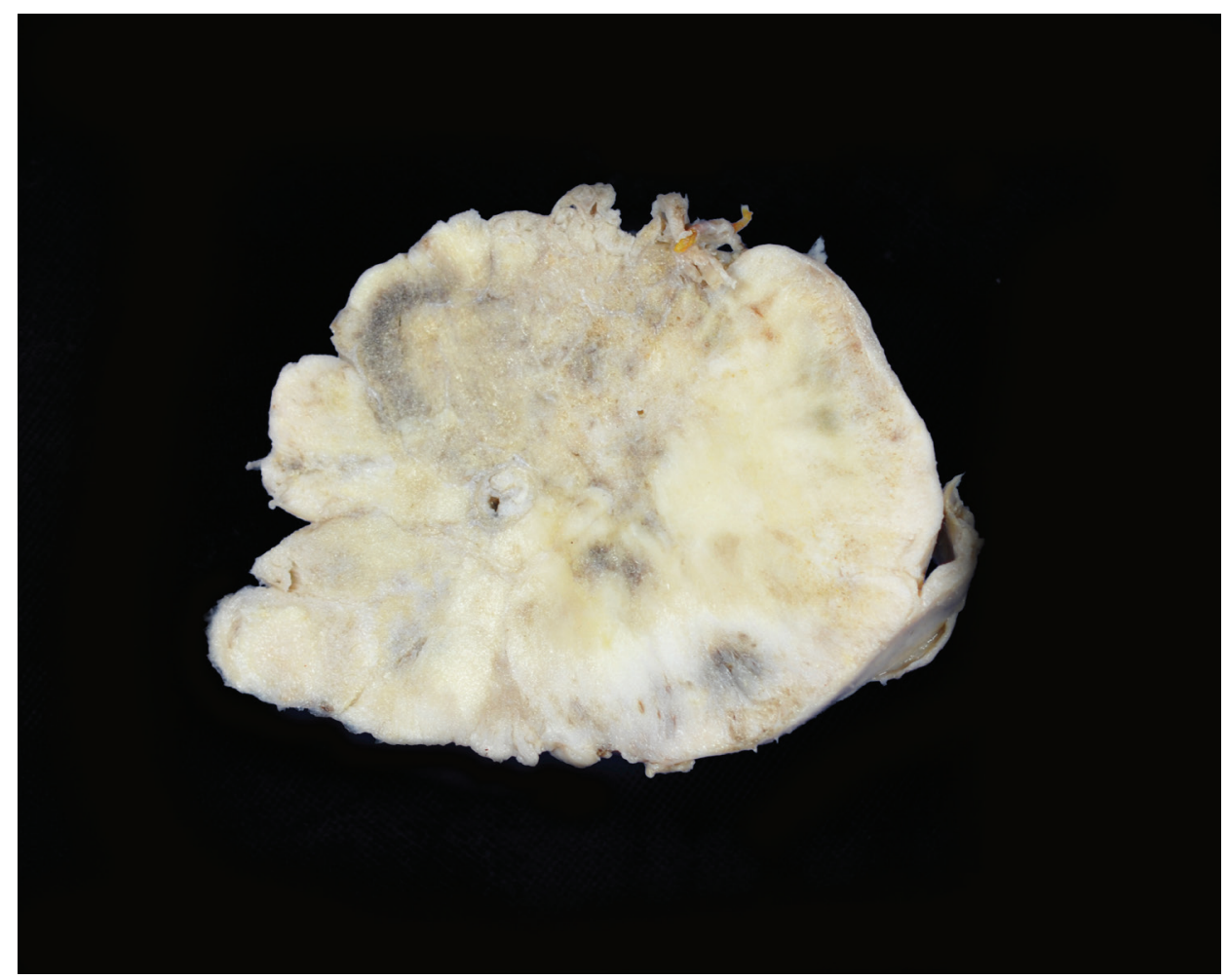

Figura 2. Osteosarcoma extraesquelético retroperitoneal, aspecto macroscópico del corte transversal del tumor de mayor tamaño $(9 \mathrm{~cm})$, de forma irregular, no encapsulado, de aspecto trabecular y consistencia dura.

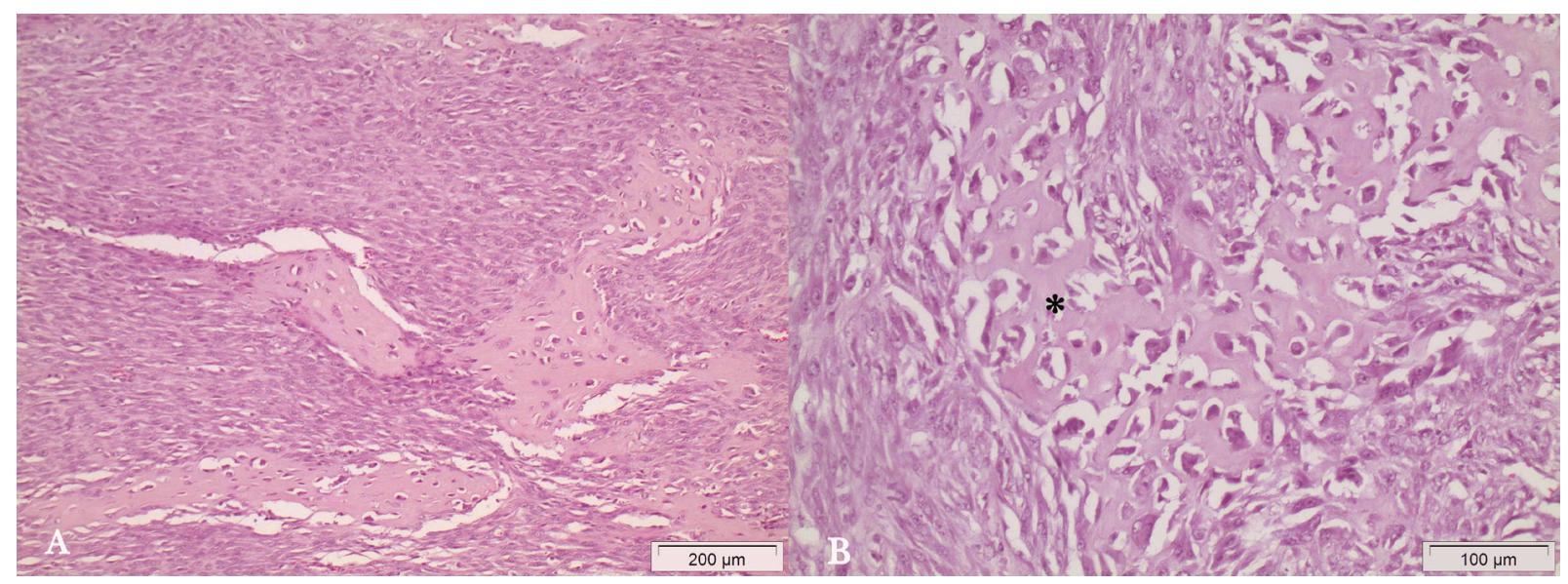

Figura 3. Osteosarcoma extraesquelético retroperitoneal, proliferación de osteoblastos con crecimiento de tipo sólido (células mesenquimales de citoplasma elongado y núcleo ovalado) con producción de osteoide (material eosinofílico amorfo) (A) H\&E 200x; osteoblastos displásicos en asociación a producción de osteoide (*) (B) H\&E (400x). 


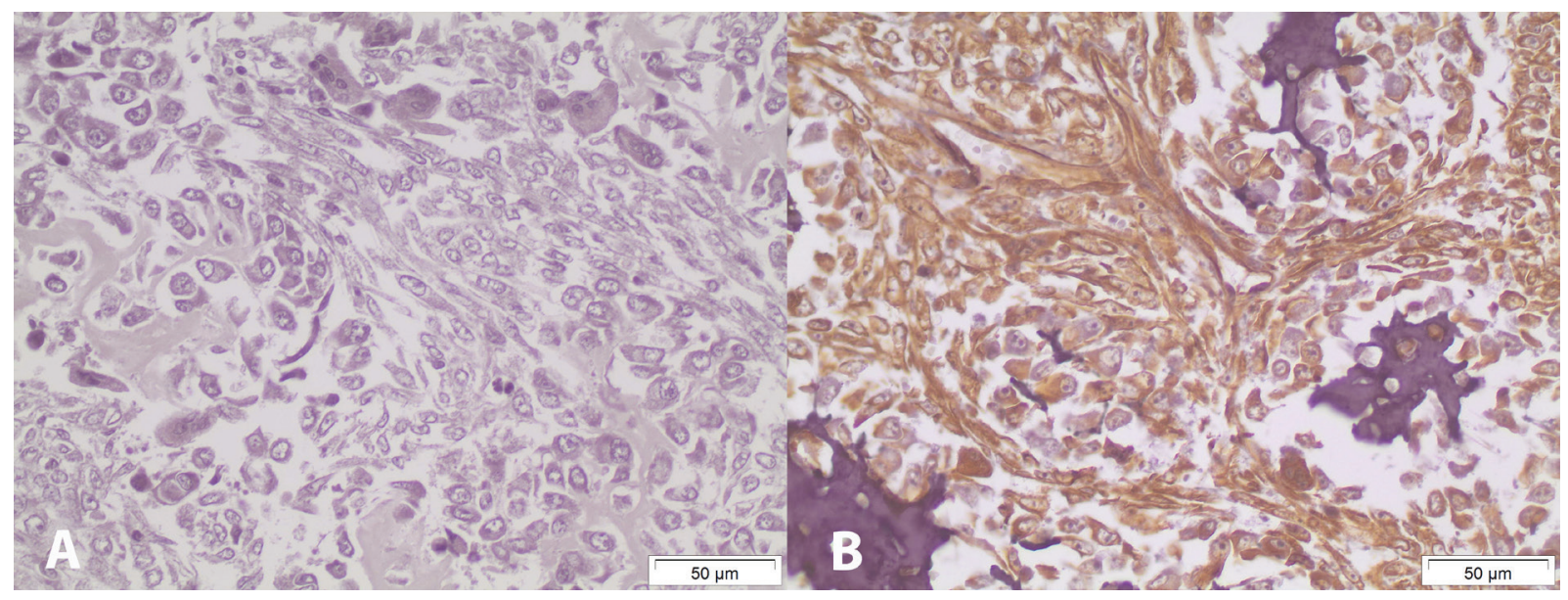

Figura 4. Osteosarcoma extraesquelético, inmnunonegatividad de los osteoblastos, osteoide y tejido de soporte para citoqueratina 400x (A), inmunopositividad para vimentina, observada como un precipitado de color café en el citoplasma de las células neoplásicas y tejido de soporte (B) 400x (IHO).

\section{Discusión}

La edad media de perros con OEE varía poco en los diferentes estudios; comúnmente se encuentra entre los 9 y los 11 años de edad (rango 2-17 años). Es decir, perros de edad avanzada (Patnaik 1990; Kuntz et al. 1998; Langenbach et al. 1998; Duffy et al. 2015), lo cual coincide con la edad media de presentación de 9 años (rango 2-13 años) de sarcomas retroperitoneales en un estudio (Liptak et al. 2004). El paciente de este reporte tenía 15 años, al momento del diagnóstico, mayor a la edad media reportada pero aún dentro del rango de edades de los estudios mencionados.

La mayoría de estudios indica que el OEE no muestra predilección de sexo, o son contradictorios al respecto (Patnaik 1990; Kuntz et al. 1998; Langenbach et al. 1998; Duffy et al. 2015).

Un estudio retrospectivo de 169 casos de OEE en perros, muestra un mayor riesgo o sobrerrepresentación de algunas razas; sin embargo, estas diferencias no eran estadísticamente significativas (Langenbach et al. 1998). Además, la mayoría de estudios indica que no hay predilección de raza, a diferencia de los osteosarcomas esqueléticos donde hay predilección por razas grandes y gigantes (Patnaik 1990; Kuntz et al. 1998; Urbiztondo et al. 2011). En un estudio retrospectivo de sarcomas retroperitoneales, la mayoría de perros eran de raza grande (Duffy et al. 2015). Sin embargo, en este estudio, el OEE no representaba la mayoría de tumores. No se ha reportado predisposición a OEE en perros de raza mixta, como en este caso.

El paciente presentó, al momento de la evaluación física hiporexia: constipación, dolor abdominal y se detectó una masa intraabdominal durante la palpación. Los signos clínicos más comunes, que presentan los pacientes con OEE, son: depresión, letargia, distensión 
abdominal, dificultad respiratoria, pirexia, pérdida de peso, dolor abdominal y signos gastrointestinales como, disminución del apetito, anorexia, vómito y melena (Liptak et al. 2004; MacKenzie et al. 2012; Timian et al. 2012). La presencia de estos signos inespecíficos en pacientes con sarcomas retroperitoneales es común; sin embargo, pueden presentarse en perros con otros tumores, incluyendo neoplasias renales, hepáticas y esplénicas (Liptak et al. 2004). Los tumores originados en el espacio retroperitoneal son delimitados por la columna vertebral, la musculatura paravertebral y los órganos abdominales; por tanto, el desarrollo de estos signos inespecíficos está relacionado con el tamaño del tumor, el cual puede comprimir órganos adyacentes, invadir tejido y provocar la distensión del peritoneo (Liptak et al. 2004).

Enlamitad deloscasos, de un estudio retrospectivo de 14 casos de sarcomas retroperitoneales, había involucramiento de estructuras anatómicamente adyacentes (Liptak et al. 2004); sin embargo, en el paciente de este reporte, ambos OEE removidos del espacio retroperitoneal no infiltraban otras estructuras. En perros, la metástasis es común, ocurre en un $64 \%$ de los casos y, usualmente, en los pulmones (Kuntz et al. 1998; Woldemeskel \& Grice 2011). Sin embargo, las imágenes diagnósticas y la examinación abdominal durante la cirugía, no evidenciaron la presencia de metástasis.

Las anormalidades del hemograma en el paciente, como leucocitosis por neutrofilia y anemia, además de anormalidades del panel de bioquímica sérica, como elevación del nitrógeno ureico y creatinina, se han observado en otros reportes de OEE (Liptak et al. 2004; Sato et al. 2004; Urbitzondo et al. 2010; Timian et al. 2011; MacKenzie et al. 2012; Marcus et al. 2012). Sin embargo, estas alteraciones son hallazgos que no se pueden atribuir totalmente a la presencia del tumor. En un reporte de sarcomas retroperitoneales en perros y en otro de un $\mathrm{OEE}$ en el pericardio de un perro, al igual que en el caso presente, se observa elevación de la actividad de la FAS, alteración que ha sido asociada con un peor pronóstico en perros con osteosarcoma apendicular (Ehrhart et al. 2013). La importancia clínica de la elevación de la actividad de la FAS en casos de OEE no se ha determinado. Los problemas renales, originados en pacientes con sarcomas retroperitoneales, se asocian con la invasión tumoral y compresión del tejido renal (Liptak et al. 2004).

En varios estudios sobre OEE, el órgano intraabdominal más comúnmente afectado es el bazo (Patnaik 1990; Kuntz et al. 1998; Langenbach et al. 1998; Duffy et al. 2015). Sin embargo, en el paciente de este reporte, la lesión en bazo fue confirmada histológicamente como hiperplasia folicular, descartando un posible origen primario esplénico del OEE. La hiperplasia nodular frecuentemente es observada en el bazo de perros de edad avanzada y toros viejos. La mayoría de los nódulos son de $2 \mathrm{~cm}$ de diámetro, e incluso pueden llegar a medir $5 \mathrm{~cm}$. Al cortar los nódulos, se puede apreciar cómo tienen una coloración blancuzca o grisácea, e histológicamente se componen de una proliferación linfocítica, no encapsulada, que comprime las trabéculas del tejido conectivo. La mayoría de las hiperplasias nodulares esplénicas no tienen relevancia clínica (Valli et al. 2016).

La decisión de realizar la eutanasia al paciente se tomó por la dificultad de la remoción completa del tejido tumoral. En muchos casos, como en el de este reporte, se opta por la 
eutanasia en pacientes con $\mathrm{OEE}$, debido a la localización intraabdominal, las pocas opciones quirúrgicas para poder retirar el tumor en algunos sitios anatómicos y el pobre estado clínico del animal (Thompson 2007; Urbiztondo et al. 2010). El OEE es un tumor extremadamente agresivo. Se estima que el tiempo de sobrevivencia, sin tratamiento, va de 23 a 33 días (Patnaik 1990; Kuntz et al. 1998; Urbiztondo et al. 2010; Timian et al. 2011; Mackenzie et al. 2012; Marcus et al 2012) a diferencia del osteosarcoma esquelético, en el cual, el tiempo de sobrevivencia, en pacientes sin quimioterapia, es de 139 a 218 días (Kuntz et al.1998). El uso de quimioterapia, en conjunto con la resección quirúrgica, aumenta la esperanza de vida a 146 días en promedio, en pacientes con OEE (Mackenzie et al. 2012); mientras que, en pacientes con osteosarcomas esqueléticos, luego de quimioterapia y resección quirúrgica, la esperanza de vida aumenta a 301-415 días (Kuntz et al. 1998). El pronóstico del OEE empeora si se localiza en la cavidad abdominal (Marcus et al. 2012) con un tiempo medio de supervivencia de 0 días, reportado en un estudio.

En conclusión, el OEE es una neoplasia mesenquimal infrecuente que no involucra, primariamente, tejido óseo y posee un pésimo pronóstico debido a su comportamiento agresivo, infiltrativo y metastásico, especialmente cuando son intraabdominales. La localización retroperitoneal del $\mathrm{OEE}$ ha sido reportada pocas veces en perros y en la literatura veterinaria en general.

\section{Referencias}

Duffy, D., Selmic, L.E., Kendall, A.R. \& Powers, B.E. 2015. Outcome Following Treatment of Soft Tissue and Visceral Extraskeletal Osteosarcoma in 33 Dogs: 2008-2013. Veterinary and Comparative Oncology. doi:10.1111/vco.12141.

Ehrhart, N.P., Ryan, S.D. \& Fan, T.M. 2013. Tumors of the Skeletal System. In: Withrow, S.J., Vail, D.M. \& Page, R.L. (Eds.). Withrow \& MacEwen's Small Animal Clinical Oncology. 5ta ed. Elsevier. China. p. 463-503.

Kuntz, C.A., Dernell, W.S., Powers, B.E. \& Withrow, S.J. 1998. Extraskeletal Osteosarcomas in Dogs: 14 Cases. Journal of the American Animal Hospital Association 34 (1): 26-30. doi: 10.5326/15473317-34-1-26

Langenbach, A., Anderson, M.A., Dambach, D.M., Sorenmo, K.U. \& Shofer, F.D. 1998. Extraskeletal Osteosarcomas in Dogs: A Retrospective Study of 169 Cases (19861996). Journal of the American Animal Hospital Association 34 (2): 113-20. doi: 10.5326/15473317-34-2-113

Liptak, J.M., Dernell, W.S., Ehrhart, E.J., Rizzo, S.A., Rooney, M.B. \& Withrow, S.J. 2004. Retroperitoneal Sarcomas in Dogs: 14 Cases (1992-2002). Journal of the American Veterinary Medical Association 224 (9): 1471-77. doi:10.2460/javma.2004.224.1471.

MacKenzie, S., Hecht, S., Sura, P.A. \& Craig, L.E. 2012. What Is Your Diagnosis ? Journal of the American Veterinary Medical Association 240 (7): 817-18. doi:10.2460/ javma.240.7.817. 
Marcus, E.C., Marolf, A., Thamm, D.H. \& Lana, S.E. 2012. What Is Your Diagnosis ? Journal of the American Veterinary Medical Association 240 (3): 265-66. doi:10.2460/ javma.240.3.265.

Miller, M.A., Aper, R.L., Fauber, A., Blevins, W.E. \& Ramos-Vara, J.A. 2006. Extraskeletal Osteosarcoma Associated with Retained Surgical Sponge in a Dog. Journal of Veterinary Diagnostic Investigation 18 (2): 224-28. doi:10.1177/104063870601800218.

Miller, M. 2016. Introduction to the Diagnostic Process in: Maxie, G. (Ed.). Jubb, Kennedy \& Palmer's Pathology of Domestic Animals, Volume 1. $6^{\text {th }}$. ed. Saunders Elsevier. China. p. 1-15.

Patnaik, A.K. 1990. Canine Extraskeletal Osteosarcoma and Chondrosarcoma: A Clinicopathologic Study of 14 Cases. Veterinary Pathology 27 (1): 46-55. doi:10.1177/030098589002700107.

Sato, T.; Koie, H.; Shibuya, H. \& Suzuki, K. 2004. Extraskeletal Osteosarcoma in the Pericardium of a Dog. The Veterinary Record 155 (24): 780-81. doi:10.1136/vr.155.24.780.

Spugnini, E.P., Ruslander, D. \& Bartolazzi, A. 2001. Extraskeletal Osteosarcoma in a Cat. Journal of the American Veterinary Medical Association 219 (1): 60-62. doi:10.2460/ javma.2001.219.60.

Thompson, K. 2007. Bones and Joints. In: Maxie, G. (Ed.). Jubb, Kennedy \& Palmer's Pathology of Domestic Animals, Volume 1. 5t $\mathrm{t}^{\mathrm{a}}$ ed. Saunders Elsevier. China. p. 1-184.

Timian, J., Yoshimoto, S.K. \& Bruyette, D.S. 2011. Extraskeletal Osteosarcoma of the Heart Presenting as Infective Endocarditis. Journal of the American Animal Hospital Association 47 (2): 129-32. doi:10.5326/JAAHA-MS-5615.

Urbiztondo, R., Chapman, S. \& Benjamino, K. 2010. Primary Mesenteric Root Osteosarcoma in a Dog. Veterinary Clinical Pathology 39 (3): 377-80. doi:10.1111/j.1939165X.2010.00239.x

Van de Sandt, R.R.O.M., Boevé, M.H., Stades, F.C., Kik, M.J.L. \& Kirpenseijn, J. 2004. Intraocular Osteosarcoma in a Dog. Journal of Small Animal Practice 45 (7): 372-74. doi:10.1111/j.1748-5827.2004.tb00251.x.

Valli, T., M. Kiupel \& D. Brienzle. 2016. Hematopoietic System in: Maxie, G. (Ed.). Jubb, Kennedy \& Palmer's Pathology of Domestic Animals, Volume 1. $6^{\text {th }}$ ed. Saunders Elsevier. China. p. $102-268$.

Woldemeskel, M.W. \& Grice, C.D. 2011. Pathology in Practice. Journal of the American Veterinary Medical Association 238 (3): 297-99. doi:10.2460/javma.238.3.297. 